K. Shikishima-Tsuji and M. Katsura

Nagoya Math. J.

Vol. 125 (1992), 93-103

\title{
HYPERTRANSCENDENTAL ELEMENTS OF A FORMAL POWER-SERIES RING OF POSITIVE CHARACTERISTIC
}

\author{
KAYOKO SHIKISHIMA-TSUJI AND MASASHI KATSURA
}

\section{§. Introduction}

Throughout this paper, we denote by $\mathbf{N}, \mathbf{Q}$ and $\mathbf{R}$ the set of all natural numbers containing 0 , the set of all rational numbers, and the set of all real numbers, respectively.

Let $K$ be a fixed field of positive characteristic $p$ and $K_{a}$ an algebraic closure of $K$. We denote by $K \llbracket X \rrbracket$ the formal power-series ring and by $d=\left(d_{\mu} ; \mu \in \mathbf{N}\right)$ the formal derivation of $K \llbracket X \rrbracket$, i.e., for every $A=\sum_{i=0}^{\infty} a_{i} X^{i}$ $\in K \llbracket X \rrbracket$, the $\mu$-th derivative $d_{n} A$ of $A$ is defined by

$$
d_{\mu} A=\sum_{\imath=\mu}^{\infty}\left(\begin{array}{l}
i \\
\mu
\end{array}\right) a_{i} X^{i-\mu} .
$$

For differential rings and differential fields of positive characteristic, see Okugawa [4].

This paper contains three theorems. Let $A$ be an element $\sum_{i=0}^{\infty} a_{i} X^{i}$ of $K \llbracket X \rrbracket$. We say that $A$ is hypertranscendental over $K$, if, for every $\mu \in \mathbf{N}, A, d_{1} A, \cdots, d_{\mu} A$ are algebraically independent over $K(X)$. When the characteristic of the field is zero, the existence of hypertranscendental elements is well-known (see D. Hilbert [1], O. Hölder [2], F. Kuiper [3]). Theorem 1 shows the existence of hypertranscendental elements in case of positive characteristic.

Let $L$ be a differential field and $S$ a subset of a differential extension field of $L$. We say that $S$ is differentially independent over $L$ or all the elements of $S$ are differentially independent over $L$, if for every $\mu \in \mathbf{N}$ and elements $s_{1}, \cdots, s_{\mu}$ of $S$, there are no nonzero differential polynomial $F\left(X_{1}, \cdots, X_{\mu}\right) \in L\left\{X_{1}, \cdots, X_{\mu}\right\}$ such that $F\left(s_{1}, \cdots, s_{\mu}\right)=0$.

Theorem 2 states that there are infinitely many hypertranscendental

Received February 21, 1991. 
elements in $K \llbracket X \rrbracket$ over $K$ which are differentially independent over $K$.

If an element of $K \llbracket X \rrbracket$ is differentially quasi-algebraic over $K$ (see K. Shikishima-Tsuji [5]), then

$$
\lim _{s \rightarrow \infty} \frac{1}{s} \operatorname{tr} \operatorname{deg}\left\{d_{\mu} A ; \mu<s\right\} / K(X)=0 .
$$

If $A$ is hypertranscendental over $K$, then

$$
\lim _{s \rightarrow \infty} \frac{1}{s} \operatorname{tr} \operatorname{deg}\left\{d_{\mu} A ; \mu<s\right\} / K(X)=1 .
$$

Let $A$ be hypertranscendental over $K$. It can be easily shown that, for every $0<r<p$, the formal power series $B=d_{p-r} A$ satisfies the equation

$$
\lim _{s \rightarrow \infty} \frac{1}{s} \operatorname{tr} \operatorname{deg}\left\{d_{\mu} B ; \mu<s\right\} / K(X)=\frac{r}{p} .
$$

For every $\alpha \in \mathbf{R}(0 \leq \alpha \leq 1)$, there exists a formal power series $B_{\alpha}$ of $K \llbracket X \rrbracket$ such that

$$
\lim _{s \rightarrow \infty} \frac{1}{s} \operatorname{tr} \operatorname{deg}\left\{d_{\mu} B_{\alpha} ; \mu<s\right\} / K(X)=\alpha .
$$

This is Theorem 3.

$\S 1$.

For $m, n \in \mathbf{N}$, the binomial coefficient $\left(\begin{array}{c}m \\ n\end{array}\right)$ equals $\frac{m !}{n !(m-n) !}$ in case $m \geq n$, otherwise zero.

LemMA 1. Let $m, n \in \mathbf{N}$. If $m=\sum_{i=0}^{e} m_{i} p^{i}$ and $n=\sum_{i=0}^{e} n_{i} p^{i}$ are the $p$-adic expressions of $m$ and $n$ respectively, then

$$
\left(\begin{array}{c}
m \\
n
\end{array}\right) \equiv\left(\begin{array}{c}
m_{0} \\
n_{0}
\end{array}\right) \cdots\left(\begin{array}{c}
m_{e} \\
n_{e}
\end{array}\right) \quad(\bmod p) .
$$

Proof. By expanding both sides of the identity over the prime field of characteristic $p$ :

$$
(1+x)^{m}=(1+x)^{m_{0}}\left(1+x^{p}\right)^{m_{1}}\left(1+x^{p^{2}}\right)^{m_{2}} \cdots\left(1+x^{p^{e}}\right)^{m_{e}},
$$

and comparing the coefficients of $x^{n}$, we obtain the congruence (1). q.e.d.

Lemma 2. Let $m, n, e, t$ be natural numbers. For $t<p^{e}$, we have the 
following statements:

(1) If $m \equiv n\left(\bmod p^{e}\right)$, then $\left(\begin{array}{c}m \\ t\end{array}\right) \equiv\left(\begin{array}{c}n \\ t\end{array}\right)(\bmod p)$.

(2) If $m \equiv r\left(\bmod p^{e}\right)$ and $0 \leq r \leq t-1$, then $\left(\begin{array}{c}m \\ t\end{array}\right) \equiv 0(\bmod p)$.

(3) If $m \equiv t\left(\bmod p^{e}\right)$, then $\left(\begin{array}{c}m \\ t\end{array}\right) \equiv 1(\bmod p)$.

Proof. (1) Let $m=\sum_{i=0}^{\alpha} m_{i} p^{i}, n=\sum_{i=0}^{\alpha} n_{i} p^{i}$ and $t=\sum_{i=0}^{\alpha} t_{i} p^{i}$ be the $p$-adic expressions of $m$ and $n$ respectively. Since $m \equiv n\left(\bmod p^{e}\right)$, we have $m_{0}=n_{0}, \cdots, m_{e-1}=n_{e-1}$. Lemma 1 implies that

$$
\begin{aligned}
\left(\begin{array}{c}
m \\
t
\end{array}\right) & \equiv\left(\begin{array}{c}
m_{0} \\
t_{0}
\end{array}\right) \cdots\left(\begin{array}{c}
m_{e-1} \\
t_{e-1}
\end{array}\right)\left(\begin{array}{c}
m_{e} \\
0
\end{array}\right) \cdots\left(\begin{array}{c}
m_{\alpha} \\
0
\end{array}\right) \\
& \equiv\left(\begin{array}{c}
n_{0} \\
t_{0}
\end{array}\right) \cdots\left(\begin{array}{c}
n_{e-1} \\
t_{e-1}
\end{array}\right)\left(\begin{array}{c}
n_{e} \\
0
\end{array}\right) \cdots\left(\begin{array}{c}
n_{\alpha} \\
0
\end{array}\right) \equiv\left(\begin{array}{c}
n \\
t
\end{array}\right)(\bmod p) .
\end{aligned}
$$

(2) Since $r \leq t-1$, we have $\left(\begin{array}{l}r \\ t\end{array}\right)=0$. By (1), we have

$$
\left(\begin{array}{c}
m \\
t
\end{array}\right) \equiv\left(\begin{array}{l}
r \\
t
\end{array}\right) \quad(\bmod p)
$$

(3) By (1), we have

$$
\left(\begin{array}{c}
m \\
t
\end{array}\right) \equiv\left(\begin{array}{l}
t \\
t
\end{array}\right) \quad(\bmod p)
$$

q.e.d.

Let $B$ be a formal power series of $K \llbracket X \rrbracket$. We denote the leading degree of $B$ by $v(B)$ (i.e., if $B=\sum_{i=r}^{\infty} b_{i} X^{i}$ and $b_{r} \neq 0$, then $v(B)=r$ and if $B=0$, then $v(B)=\infty$ ).

TheOREM 1. Let $A$ be an element $\sum_{i=0}^{\infty} a_{i} X^{m_{i}}$ of $K \llbracket X \rrbracket$ with nonzero $a_{i} \in K(i \in \mathbf{N})$ and $m_{0}<m_{1}<m_{2}<\cdots$ be natural numbers. If $A$ satisfies the following condition, then $A$ is hypertranscendental over $K$.

For any $e, s \in \mathbf{N}$, there exist natural numbers $i_{0}<i_{1}<i_{2}<\cdots$ such that

$$
m_{i_{j}} \equiv s\left(\bmod p^{e}\right) \quad \text { and } \lim _{j \rightarrow \infty} \frac{m_{i_{j}}}{m_{i_{j}-1}}=\infty .
$$

Proof. Suppose $A$ is not hypertranscendental over $K_{a}$. Then, there is a positive integer $\mu$ such that $A, d_{1} A, \cdots, d_{\mu} A$ are algebraically dependent over $K_{a}(X)$, that is, there exists a non-zero polynomial $F\left(X, Y_{0}, \cdots, Y_{\mu}\right)$ $\in K_{a}\left[X, Y_{0}, \cdots, Y_{\mu}\right]$ which satisfies the following two conditions: 
(2) $F\left(X, A, d_{1} A, \cdots, d_{\mu} A\right)=0$.

(3) If $G\left(X, Y_{0}, \cdots, Y_{\mu}\right)$ is non-zero polynomial such that $G\left(X, A, d_{1} A, \cdots\right.$, $\left.d_{\mu} A\right)=0$, then the total degree of $G$ is not smaller than that of $F$.

We see that $F$ is irreducible by the condition (3).

Let $c_{1}$ and $c_{2}$ be the degrees of $F$ on $X$ and on $Y_{0}, \ldots, Y_{\mu}$, respectively. We take a natural number $e$ such that $\mu<p^{e}$. By the assumption (1), there exist $k_{0}, k_{1}, \cdots, k_{\mu} \in \mathbf{N}$ such that the following conditions hold for every $s(0 \leq s \leq \mu)$;

(4) $m_{k_{s}-1} \geq c_{1}+\mu$,

(5) $m_{k_{s}}>\left(c_{2}+1\right) m_{k_{s}-1}$,

(6) $m_{k_{s}} \equiv s\left(\bmod p^{e}\right)$, and,

(7) $m_{k_{s}}>v\left(\frac{\partial F}{\partial Y_{t}}\left(X, A, d_{1} A, \cdots, d_{\mu} A\right)\right)+2 \mu$, for every $t(0 \leq t \leq \mu)$ such that $\frac{\partial F}{\partial Y_{\iota}}\left(X, A, d_{1} A, \cdots, d_{\mu} A\right) \neq 0$.

Let

$$
G_{s}=\sum_{i=0}^{k_{s}-1} a_{i} X^{m_{i}} \quad \text { and } \quad B_{s}=\sum_{i=k_{s}}^{\infty} a_{i} X^{m_{i}} \quad(0 \leq s \leq \mu) .
$$

By Taylor's expansion, we have

$$
\begin{aligned}
0 & =F\left(X, A, d_{1} A, \cdots, d_{\mu} A\right) \\
& =F\left(X, G_{s}, d_{1} G_{s}, \cdots, d_{\mu} G_{s}\right)+\sum_{t=0}^{\mu} d_{t} B_{s} \frac{\partial F}{\partial Y_{t}}\left(X, A, d_{1} A, \cdots, d_{\mu} A\right)-E_{s}
\end{aligned}
$$

where $E_{s}$ is a sum of terms of degree $\geq 2$ in $\left\{B_{s}, d_{1} B_{r}, \cdots, d_{\mu} B_{s}\right\}$. We have

$$
\begin{aligned}
& \operatorname{deg} F\left(X, G_{s}, d_{1} G_{s}, \cdots, d_{n} G_{s}\right) \leq c_{1}+c_{2} m_{k_{s}-1}, \\
& v\left(d_{t} B_{s} \frac{\partial F}{\partial Y_{t}}\left(X, A, d_{1} A, \cdots, d_{\mu} A\right)\right) \geq v\left(d_{t} B_{s}\right) \geq m_{k_{s}}-t,
\end{aligned}
$$

and

$$
v\left(E_{s}\right) \geq \min _{0 \leq t_{1}, t_{2} \leq \mu}\left\{v\left(d_{t_{1}} B_{s} d_{t_{2}} B_{s}\right)\right\} \geq 2\left(m_{k_{s}}-\mu\right) .
$$

Hence, by (4) and (5), we have

$$
\begin{aligned}
v\left(\sum_{t=0}^{\mu} d_{t}\right. & \left.B_{s} \frac{\partial F}{\partial Y_{t}}\left(X, A, d_{1} A, \cdots, d_{\mu} A\right)-E_{s}\right) \\
& \geq m_{k_{s}}-\mu>\left(c_{2}+1\right) m_{k_{s}-1}-\mu \\
& \geq c_{2} m_{k_{s}-1}+c_{1} \geq \operatorname{deg} F\left(X, G_{s}, d_{1} G_{s}, \cdots, d_{\mu} G_{s}\right)
\end{aligned}
$$


Therefore, $F\left(X, G_{s}, d_{1} G_{s}, \cdots, d_{\mu} G_{s}\right)=0$ and

$$
\sum_{t=0}^{\mu} d_{t} B_{s} \frac{\partial F}{\partial Y_{t}}\left(X, A, d_{1} A, \cdots, d_{\mu} A\right)=E_{s} \quad(s=0,1, \cdots, \mu) .
$$

Let

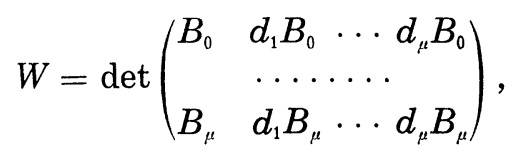

and

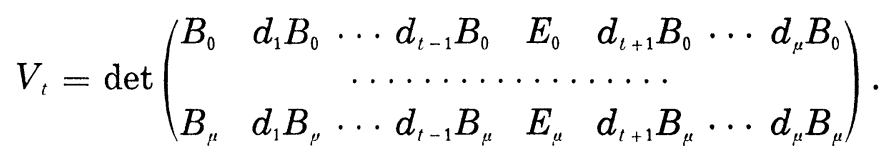

On the other hand, $d_{t} B_{s}=\sum_{i=k_{s}}^{\infty}\left(\begin{array}{c}m_{i} \\ t\end{array}\right) a_{i} X^{m_{i-t}}$, and by (6) and Lemma 2,

$$
\left(\begin{array}{c}
m_{k_{s}} \\
s
\end{array}\right)=1, \quad\left(\begin{array}{c}
m_{k_{s}} \\
s+1
\end{array}\right)=\cdots=\left(\begin{array}{c}
m_{k_{s}} \\
\mu
\end{array}\right)=0 .
$$

Hence, the coefficient of the leading form of the power series $W$ is $a_{k_{0}} \cdots a_{k_{\mu}}$ and $v(W)=m_{k_{0}}+\cdots+m_{k_{\mu}}-\frac{\mu(\mu+1)}{2}$. Therefore, $W \neq 0$. By Cramer's rule, (9) implies

$$
W \frac{\partial F}{\partial Y_{t}}\left(X, A, d_{1} A, \cdots, d_{\mu} A\right)=V_{t}
$$

We have

$$
\begin{aligned}
v\left(V_{t}\right) & \geq \min _{0 \leq s \leq \nu}\left\{\left(m_{k_{0}}+\cdots+m_{k_{\mu}}-\frac{\mu(\mu+1)}{2}\right)-\left(m_{k_{s}}-t\right)+v\left(E_{s}\right)\right\} \\
& \geq v(W)+\min _{0 \leq s \leq \mu}\left\{v\left(E_{s}\right)-m_{k_{s}}\right\} .
\end{aligned}
$$

If $\frac{\partial F}{\partial Y_{t}}\left(X, A, d_{1} A, \cdots, d_{\mu} A\right) \neq 0$, then by (7), (8) and (10), we have

$$
\begin{gathered}
v\left(\frac{\partial F}{\partial Y_{t}}\left(X, A, d_{1} A, \cdots, d_{\mu} A\right)\right)=v\left(V_{t}\right)-v(W) \\
\geq \min _{0 \leq s \leq \mu}\left\{v\left(E_{s}\right)-m_{k_{s}}\right\} \\
\geq \min _{0 \leq s \leq \mu}\left\{m_{k_{s}}-2 \mu\right\}>v\left(\frac{\partial F}{\partial Y_{t}}\left(X, A, d_{1} A, \cdots, d_{\mu} A\right)\right),
\end{gathered}
$$

which is a contradiction. Therefore, we have 


$$
\frac{\partial F}{\partial Y_{t}}\left(X, A, d_{1} A, \cdots, d_{\mu} A\right)=0 \quad(0 \leq t \leq \mu) .
$$

By the assumption (3), we have

$$
\frac{\partial F}{\partial Y_{t}}\left(X, Y_{0}, \cdots, Y_{\mu}\right)=0 \quad(0 \leq t \leq \mu) .
$$

Since $F$ is irreducible, it follows that $F\left(X, Y_{0}, \cdots, Y_{\mu}\right) \in K_{a}\left[X, Y_{0}^{p}, \cdots, Y_{\mu}^{p}\right]$ and there exist $F_{0}, \cdots, F_{p-1} \in K_{a}\left[X^{p}, Y_{0}^{p}, \cdots, Y_{\mu}^{p}\right]$ such that

$$
\begin{gathered}
F\left(X, Y_{0}, \cdots, Y_{\mu}\right)=F_{0}\left(X, Y_{0}, \cdots, Y_{\mu}\right)+X F_{1}\left(X, Y_{0}, \cdots, Y_{\mu}\right) \\
+\cdots+X^{p-1} F_{p-1}\left(X, Y_{0}, \cdots, Y_{\mu}\right) .
\end{gathered}
$$

Since $F\left(X, d_{1} A, \cdots, d_{\mu} A\right)=0$ and $F_{i}\left(X, d_{1} A, \cdots, d_{\mu} A\right) \in K_{a} \llbracket X^{p} \rrbracket(i=0, \cdots$, $p-1)$, we have

$$
F_{i}\left(X, d_{1} A, \cdots, d_{\mu} A\right)=0 \quad(i=0, \cdots, p-1) .
$$

Since $K_{a}$ is perfect, there exist $G_{0}, \cdots, G_{p-1} \in K_{a}\left[X, Y_{0}, \cdots, Y_{\mu}\right]$ such that

$$
F_{i}\left(X, Y_{0}, \cdots, Y_{p}\right)=\left(G_{i}\left(X, Y_{0}, \cdots, Y_{p}\right)\right)^{p} \quad(i=0, \cdots, p-1) .
$$

Since $G_{i}\left(X, d_{1} A, \cdots, d_{\mu} A\right)=0(i=0, \cdots, p-1)$, (3) implies that

$$
G_{i}\left(X, Y_{0}, \cdots, Y_{\mu}\right)=0 \quad(i=0, \cdots, p-1) .
$$

It follows that $F\left(X, Y_{0}, \cdots, Y_{\mu}\right)=0$. This is a contradiction.

q.e.d.

By this theorem, the power series

$$
\sum_{i=0}^{\infty} X^{p^{i^{2}+i}}, \quad \sum_{i=0}^{\infty} X^{i^{i} p+i} \text { and } \sum_{i=0}^{\infty} X^{i !+i}
$$

are hypertranscendental.

\section{§.}

Let $A=\sum_{i=0}^{\infty} a_{i} X^{i}$ be a formal power series of $K \llbracket X \rrbracket$. For $e \in \mathbf{N}$ and $k \in\left\{0,1, \cdots, p^{e}-1\right\}$ we denote the power series $\sum_{i=0}^{\infty} a_{k+i p^{e}} X^{i p^{e}}$ by $A_{k}^{(e)}$. Then, $A_{0}^{(e)}, \cdots, A_{p^{e}-1}^{(e)}$ are elements of $K \llbracket X^{p^{e}} \rrbracket$ and we have

$$
A=A_{0}^{(e)}+X A_{1}^{(e)}+\cdots+X^{p^{e}-1} A_{p^{e}-1}^{(e)} .
$$

Theorem 2. Let $A=\sum_{i=1}^{\infty} a_{i} X^{i}$ be hypertranscendental. For each $t(t=1, \cdots, p-1)$ and $s \in \mathbf{N}-\{0\}$, let

$$
B_{s, t}=\left(A_{t p^{s-1}}^{(s)}\right)^{p-s}=\sum_{i=0}^{\infty}\left(a_{t p^{s-1}+i p^{s}}\right)^{p-s} X^{i}
$$


Then, $\left\{B_{s, t} ; s \in \mathbf{N}-\{0\}, t=1, \cdots, p-1\right\}$ are differentially independent over $K_{a}(X)$.

Remark. Let $m_{0}<m_{1}<m_{2}<\ldots$ be a sequence of natural numbers satisfying the condition (1) of Theorem 1 . The power series $A=\sum_{i=0}^{\infty} a_{i} X^{i}$ where $a_{i}=1$ if $i$ equals some $m_{j}(j \in \mathbf{N})$, otherwise 0 , is hypertranscendental over $K$ by Theorem 1 . Therefore, by Theorem $2, B_{s, t}=\sum_{i=0}^{\infty} a_{t p^{s-1}+i p^{s}} X^{i}$ $(s \in \mathbf{N}-\{0\}, t=1, \cdots, p-1)$ are differentially independent over $K(X)$.

Proof of Theorem 2. By $A_{0}^{(s-1)}=\sum_{t=0}^{p-1} X^{t p^{s-1}} A_{t p^{s-1}}^{(s)}$, we have

$$
A=A_{0}^{(e)}+\sum_{s=1}^{e} \sum_{t=1}^{p-1} X^{t p^{s-1}} A_{t p^{s-1}}^{(s)} .
$$

Hence, for $1 \leq \mu \leq p^{e}-1$,

$$
d_{\mu} A=d_{\mu} A_{0}^{(e)}+\sum_{s=1}^{e} \sum_{t=1}^{p-1} \sum_{v_{1}+v_{2}=\mu} d_{v_{1}} X^{t p^{s-1}} d_{v_{2}} A_{t p^{s-1}}^{(s)} .
$$

For every $u, d_{v} A_{u}^{(r)} \neq 0$ implies $p^{r} \mid v$. Then, $d_{\mu} A \in K\left(X, d_{v p^{s}} A_{t p^{s-1}}^{(s)} ; s=1\right.$, $\left.2, \cdots, e, t=1,2, \cdots, p-1, v=0,1, \cdots, p^{e-s}-1\right)$. Hence,

$K\left(X, d_{\mu} A ; \mu=1,2, \cdots, p^{e}-1\right)$

$$
\subseteq K\left(X, d_{v p^{s}} A_{t p^{s-1}}^{(s)} ; s=1,2, \cdots, e, t=1,2, \cdots, p-1, v=0,1, \cdots, p^{e-s}-1\right) .
$$

Since $A$ is hypertranscendental,

$\operatorname{tr} \operatorname{deg}\left\{d_{v p^{s}} A_{t p^{s-1}}^{(s)} ; s=1,2, \cdots, e, t=1,2, \cdots, p-1, v=1,2, \cdots\right.$,

$$
\begin{aligned}
p^{e-s}- & 1\} / K_{a}(X) \\
& \geq \operatorname{tr} \operatorname{deg}\left\{d_{\mu} A ; \mu=1,2, \cdots, p^{e}-1\right\} / K_{a}(X)=p^{e}-1 .
\end{aligned}
$$

However, the cardinality of the set $\{(s, t, v) ; s=1,2, \cdots, e, t=1,2, \cdots$, $\left.p-1, v=0,1, \cdots, p^{e-s}-1\right\}$ is $(p-1)\left(p^{e-1}+p^{e-2}+\cdots+p+1\right)=p^{e}-1$. Hence, $\left\{d_{v p^{s}} A_{t p^{s-1}}^{(s)} ; s=1,2, \cdots, e, t=1,2, \cdots, p-1, v=0,1, \cdots, p^{e-s}-1\right\}$ are algebraically independent over $K_{a}(X)$. Since, $d_{v p^{s}} A_{t p^{s-1}}^{(s)}=d_{v p^{s}}\left(B_{s, t}\right)^{p^{s}}$ $=\left(d_{v} B_{s, t}\right)^{p^{s}}$, we see that

$$
\left\{d_{v} B_{s, t} ; s=1,2, \cdots, e, t=1,2, \cdots, p-1, v=0,1, \cdots, p^{e-s}-1\right\}
$$

are algebraically independent over $K_{a}(X)$. Thus, we have the conclusion. q.e.d.

$\S 3$.

For $k \in \mathbf{N}$, we associate the real number $\langle\langle k\rangle$ as follows: If

$$
k=k_{0}+k_{1} p+\cdots+k_{e-1} p^{e-1} \quad\left(0 \leq k_{i} \leq p-1\right)
$$

is the $p$-adic expression, then 


$$
\langle k\rangle\rangle=\frac{k_{0}}{p}+\frac{k_{1}}{p^{2}}+\cdots+\frac{k_{e-1}}{p^{e}} .
$$

For a set $S$, the cardinal number of $S$ is denoted by $\# S$.

Lemma 3. Let $\alpha \in \mathbf{R}(0 \leq \alpha \leq 1)$. Then,

$$
\left.\lim _{s \rightarrow \infty} \frac{1}{s} \sharp\{\lambda \in \mathbf{N} ; \lambda \leq s-1,\langle\lambda\rangle\rangle \leq \alpha\right\}=\alpha \quad(s \in \mathbf{N}) .
$$

Proof. Let $\alpha=\frac{\alpha_{0}}{p}+\frac{\alpha_{1}}{p^{2}}+\cdots$ be the $p$-adic expression of $\alpha$, where there is no $n$ such that $\alpha_{n}=\alpha_{n+1}=\cdots=p-1$. We fix a natural number $s$ and associate $e=e(s) \in \mathbf{N}$ with $s$ by $p^{e-1} \leq s<p^{e}$. The set

$$
\{\lambda \in \mathbf{N} ; \lambda \leq s-1,\langle\lambda\rangle\rangle\left\langle\frac{\alpha_{0}}{p}+\cdots+\frac{\alpha_{e-1}}{p^{e}}\right\}
$$

is the disjoint union of the following sets:

$$
\begin{aligned}
T_{i j}=\{\lambda= & \lambda_{0}+\lambda_{1} p+\cdots+\lambda_{e-1} p^{e-1} ; \lambda_{0}=\alpha_{0}, \lambda_{1}=\alpha_{1}, \cdots, \lambda_{i-1}=\alpha_{i-1}, \\
& \left.\lambda_{i}=j, \lambda \leq s-1\right\} \quad\left(i=0,1, \cdots, e-1, j=0,1, \cdots, \alpha_{i}-1\right) .
\end{aligned}
$$

Let $s=s_{0}+s_{1} p+\cdots+s_{e-1} p^{e-1}$ be the $p$-adic expressions of $s$. If $\alpha_{0}+\alpha_{1} p+\cdots+\alpha_{i-1} p^{i-1}+j p^{i}<s_{0}+s_{1} p+\cdots s_{i} p^{i}$, then

$$
\# T_{i j}=s_{i+1}+s_{i+2} p+\cdots+s_{e-1} p^{e-i-2} .
$$

If $\alpha_{0}+\alpha_{1} p+\cdots+\alpha_{i-1} p^{i-1}+j p^{i} \geq s_{0}+s_{1} p+\cdots+s_{i} p^{i}$, then

$$
\# T_{i j}=s_{i+1}+s_{i+2} p+\cdots+s_{e-1} p^{e-t-2}-1 .
$$

In any case, we have

$$
\frac{s}{p^{i+1}}-1 \leq \# T_{i j} \leq \frac{s}{p^{i+1}} .
$$

It follows that

$$
\begin{aligned}
s(\alpha- & \left.\frac{1}{p^{e(s)}}\right)-(p-1) e(s) \\
& \leq s\left(\frac{\alpha_{0}}{p}+\frac{\alpha_{1}}{p^{2}}+\cdots+\frac{\alpha_{e-1}}{p^{e}}\right)-\left(\alpha_{0}+\alpha_{1}+\cdots+\alpha_{e-1}\right) \\
& =\alpha_{0}\left(\frac{s}{p}-1\right)+\alpha_{1}\left(\frac{s}{p^{2}}-1\right)+\alpha_{e-1}\left(\frac{s}{p^{e}}-1\right) \\
& \leq \sum_{i=0}^{e-1} \sum_{j=0}^{a_{i j}-1} \# T_{i j}
\end{aligned}
$$




$$
\begin{aligned}
& \leq \#\left\{\lambda \in \mathbf{N} \mid \lambda \leq s-1,\langle\lambda \lambda\rangle\left\langle\frac{\alpha_{0}}{p}+\frac{\alpha_{1}}{p^{2}}+\cdots+\frac{\alpha_{e-1}}{p^{e}}\right\}\right. \\
& \leq \#\{\lambda \in \mathbf{N}|\lambda \leq s-1,\langle\lambda\rangle\rangle \leq \alpha\} \\
& \leq \#\left\{\lambda \in \mathbf{N} \mid \lambda \leq s-1,\left\langle\langle\lambda\rangle\left\langle\frac{\alpha_{0}}{p}+\frac{\alpha_{1}}{p^{2}}+\cdots+\frac{\alpha_{e-1}}{p^{e}}\right\}+1\right.\right. \\
& \leq \sum_{i=0}^{e-1} \sum_{j=0}^{\alpha_{i}-1} \# T_{i j}+1 \\
& \leq \alpha_{0} \frac{s}{p}+\alpha_{1} \frac{s}{p^{2}}+\alpha_{e-1} \frac{s}{p^{e}}+1 \\
& =s\left(\frac{\alpha_{0}}{p}+\frac{\alpha_{1}}{p^{2}}+\cdots+\frac{\alpha_{e-1}}{p^{e}}\right)+1 \\
& \leq s \alpha+1 .
\end{aligned}
$$

Since $\lim _{s \rightarrow \infty}\left(\alpha-\frac{1}{p^{e(s)}}-\frac{(p-1) e(s)}{s}\right)=\lim _{s \rightarrow \infty}\left(\alpha+\frac{1}{s}\right)=\alpha$, we have the conclusion.

q.e.d.

Lemma 4. A power series $A$ is hypertranscendental over $K$ if and only if $\left\{A_{0}^{(e)}, \cdots, A_{p^{e}-1}^{(e)}\right\}$ is algebraically independent over $K(X)$ for every $e \in \mathbf{N}$.

Proof. It is easy to see that if $\mu \leq p^{e}-1$, then

$$
d_{\mu} A_{k}^{(e)}=d_{\mu}\left(\sum_{i=0}^{\infty} a_{k+i p^{e}} X^{i p^{e}}\right)=0
$$

for $k \in\{0,1, \cdots, \mu\}$. Since $A=A_{0}^{(e)}+X A_{1}^{(e)}+\cdots+X^{p^{e-1}} A_{p^{e}-1}^{(e)}$, the vector space spanned by $A, X d_{1} A, \cdots, X^{p^{e-1}} d_{p^{e-1}} A$ over $K$ coincides with the vector space spanned by $A_{0}^{(e)}, X A_{1}^{(e)}, \cdots, X^{p^{e}-1} A_{p^{e}-1}^{(e)}$ over $K . \quad$ q.e.d.

Theorem 3. For any $\alpha \in \mathbf{R}(0 \leq \alpha \leq 1)$, there exists a formal power series $B$ of $K \llbracket X \rrbracket$ such that

$$
\lim _{s \rightarrow \infty} \frac{1}{s} \operatorname{tr} \operatorname{deg}\left\{B, d_{1} B, \cdots, d_{s-1} B\right\} / K(X)=\alpha \quad(s \in \mathbf{N}) .
$$

Proof. Let $A=\sum_{i=0}^{\infty} a_{i} X^{i}$ be hypertranscendental. We consider the formal power series $B=\sum_{i=0}^{\infty} \varepsilon_{i} a_{i} X^{i}$ with $\varepsilon_{i}=0$ if $\left.\langle i\rangle\right\rangle>\alpha$ and $\varepsilon_{i}=1$ if $\langle i\rangle\rangle \leq \alpha$. Let $\alpha=\frac{\alpha_{0}}{p}+\frac{\alpha_{1}}{p^{2}}+\cdots$ be the $p$-adic expression of $\alpha$, where there is no $n$ such that $\alpha_{n}=\alpha_{n+1}=\cdots=p-1$. We fix a natural number $s$ and associate

$$
\begin{aligned}
& e=e(s) \in \mathbf{N} \text { by } p^{e-1} \leq s<p^{e}, \\
& t=\alpha_{0}+\alpha_{1} p+\cdots+\alpha_{e-1} p^{e-1}
\end{aligned}
$$


and

$$
\beta=\left\langle\langle t\rangle=\frac{\alpha_{0}}{p}+\frac{\alpha_{1}}{p^{2}}+\cdots+\frac{\alpha_{e-1}}{p^{e}} .\right.
$$

For every $k \in \mathbf{N}\left(k<p^{e}\right)$ such that $\left.\langle k\rangle\right\rangle>\alpha$ and every $i \in \mathbf{N}$, we have $\left.\left\langle\left\langle i p^{e}+k\right\rangle\right\rangle \geq\langle k k\rangle\right\rangle \alpha$. By the definition of $B$, we have

$$
B_{k}^{(e)}=\sum_{\imath=0}^{\infty} \varepsilon_{k+i p^{e}} a_{k+i p^{e}} X^{i p^{e}}=0 .
$$

Therefore,

(1) if $\langle\langle k\rangle\rangle>\alpha$ then $B_{k}^{(e)}=0$.

For each $j \in \mathbf{N}\left(j<p^{e}\right)$, either $\left.\langle k k\rangle\right\rangle\langle\langle j\rangle\rangle$ or else $\langle\langle j\rangle\rangle>\alpha$. In the former case, we have $\left(\begin{array}{l}j \\ k\end{array}\right)=0$ by Lemma 1 . In the latter case, we have $B_{j}^{(e)}=0$ by (1). Hence we have

$$
d_{k} B=\sum_{j=k}^{p^{e-1}}\left(\begin{array}{l}
j \\
k
\end{array}\right) B_{j}^{(e)} X^{j-k}=0
$$

Therefore,

(2) if $\langle\langle k\rangle\rangle>\alpha$ then $d_{k} B=0$.

It follows that

$$
K\left(X, B, d_{1} B, d_{2} B, \cdots, d_{s-1} B\right)=K(X)\left(d_{k} B ; k \leq s-1,\langle k\rangle \leq \alpha\right) .
$$

Hence

(3) $\operatorname{tr} \operatorname{deg}\left\{B, d_{1} B, d_{2} B, \cdots, d_{s-1} B\right\} / K(X) \leq \#\{k \in \mathbf{N} ; k \leq s-1,\langle\langle k\rangle \leq \alpha\}$.

For every $k \in \mathbf{N}\left(k<p^{e}\right)$ such that $\langle k k\rangle<\beta$ and every $i \in \mathbf{N}$, we have $\left\langle\left\langle i p^{e}+k\right\rangle\right\rangle\langle\langle k\rangle\rangle+\frac{1}{p^{e}} \leq \alpha$. By the definition of $B$, we have

$$
B_{k}^{(e)}=\sum_{i=0}^{\infty} \varepsilon_{k+i p^{e}} a_{k+i p^{e}} X^{i p^{e}}=A_{k}^{(e)} .
$$

Therefore,

(4) if $\left\langle\langle k\rangle\left\langle\beta\right.\right.$ then $B_{k}^{(e)}=A_{k}^{(e)}$.

For any $k \in \mathbf{N}$ with $k<p^{e}$ and $\langle\langle k\rangle<\beta$ it follows from (1) and (4) that

$$
d_{k} B=A_{k}^{(e)}+\left(\begin{array}{l}
t \\
k
\end{array}\right) B_{t}^{(e)} X^{t-k}+\sum\left(\begin{array}{l}
i \\
k
\end{array}\right) A_{i}^{(e)} X^{i-k}
$$

where the summation ranges over all $i$ with $k<i<p^{e}$, $\langle i i\rangle<\beta$. Therefore, 


$$
\begin{array}{r}
K\left(X, B_{t}^{(e)}\right)\left(d_{k} B ; k \leq s-1, k \neq t\right)\left(A_{i}^{(e)} ; s \leq i<p^{e},\langle\langle i\rangle\rangle<\beta\right) \\
=K\left(X, B_{t}^{(e)}\right)\left(A_{k}^{(e)} ; k<p^{e},\langle\langle k\rangle\langle\beta) .\right.
\end{array}
$$

Since $\left.\left\{A_{k}^{(e)} ; 0 \leq k<p^{e},\langle k\rangle\right\rangle<\beta\right\}$ is algebraically independent over $K(X)$ by Lemma 4 , we have

$$
\begin{aligned}
\operatorname{tr} \operatorname{deg}\{B, & \left.d_{1} B, d_{2} B, \cdots, d_{s-1} B\right\} / K(X) \\
& \geq \operatorname{tr} \operatorname{deg}\left\{d_{k} B \mid k \leq s-1, k \neq t\right\} / K\left(X, B_{t}^{(e)}\right) \\
& \geq \#\{k \in \mathbf{N} ; k \leq s-1,\langle\langle k\rangle\rangle\langle\beta\}-1 .
\end{aligned}
$$

Since $\{k \in \mathbf{N} ; k \leq s-1,\langle k\rangle\rangle\langle\beta\}=\{k \in \mathbf{N} ; k \leq s-1,\langle\langle k\rangle \leq \alpha\}-\{t\}$, we have

$$
\begin{aligned}
\operatorname{tr} \operatorname{deg}\left\{B, d_{1} B, d_{2} B, \cdots, d_{s-1} B\right\} / K(X) \\
\geq \#\{k \in \mathbf{N} ; k \leq s-1,\langle\langle k\rangle \leq \alpha\}-2 .
\end{aligned}
$$

Now the conclusion follows from (3), (5) and Lemma 3.

q.e.d.

The problem treated in this paper has been derived from a subject suggested by Professor Hideyuli Matsumura, for whom the authors are grateful.

\section{REFERENCES}

[1] D. Hilbert, Mathematische Probleme, C.R. du 2. congrès international des math., Paris 1902.

[ 2 ] O. Hölder, Ueber die Eigenschaft der Gammafunction keiner algebraischen Differentialgleichung zu genügen, Math. Ann., 28 (1886), 1-13.

[ 3 ] F. Kuiper, On algebraic independence in differential rings, Proc. Kon. Ned. Akad. Wetensch., Ser. A67 (1964), 90-103.

[ 4 ] K. Okugawa, "Differential Algebra of Nonzero Characteristic", Lectures in Mathematics, Department of Math. Kyoto Univ., 16, 1987.

[5] K. Shikishima-Tsuji, Differentially quasi-algebraic extension of positive characteristic $p$, submitted.

Kayoko Shikishima-Tsuji

Institute of Mathematics

Yoshida College

Kyoto University

Kyoto 606, Japan

Masashi Katsura

Department of Mathematics

Faculty of Science

Kyoto Sangyo University

Kyoto 603, Japan 